International Electronic Journal of Geometry

Volume 7 No. 2 PP. 92-100 (2014) (C)IEJG

\title{
THE KV-COHOMOLOGY AND LOCALLY FLAT HYPERSURFACES
}

\author{
ALAIN MUSESA LANDA
}

(Communicated by Murat TOSUN)

\begin{abstract}
We show that the second fundamental form of locally flat hypersurfaces defines under certain conditions, a cohomology class in the KoszulVinberg complex of the multilinear maps from the algebra of smooth vector fields on the hypersurface with values in the space of smooth functions of the manifold. We discuss the geometric interpretation of the triviality of this class for convex locally flat hypersurfaces.
\end{abstract}

\section{INTRODUCTION}

An algebra $\mathbb{A}$ over a commutative field $\mathbb{F}$ with characteristic zero is called a Koszul-Vinberg algebra if for all $a, b, c \in \mathbb{A}, a(b c)-(a b) c=b(a c)-(b a) c$. KoszulVinberg algebras generalize associative algebras. In his paper [6], M.Nguiffo Boyom defined an intrinsic cohomology theory for Koszul-Vinberg algebras and their modules. A locally flat manifold is a triple $(M, g, D)$ where $(M, g)$ is a Riemannian manifold and $D$ a linear torsion-free connection whose curvature tensor vanishes identically. The vector space $\mathfrak{X}(M)$ of smooth vector fields on $(M, g, D)$ possesses a natural structure of real Koszul-Vinberg algebra, under the multiplication $X Y=D_{X} Y$ for all $X, Y \in \mathfrak{X}(M)$. We will denote it by $\mathbb{A}=(\mathfrak{X}(M), D)$. Moreover, the algebra $\mathbb{A}=(\mathfrak{X}(M), D)$ acts on the module $C^{\infty}(M)$ by the map $X . f=d f(X)$, for all $X \in \mathfrak{X}(M)$ and for all $f \in C^{\infty}(M)$.

In this note, we use the Koszul-Vinberg cohomology as a tool to study locally flat hypersurfaces of Riemannian manifolds. The main purpose of the present work is to show that the scalar second fundamental form $A$ of a locally flat hypersurface $N$ of a Riemannian manifold $(M, g)$, under certain conditions, difines a KV-cohomology class $A \in H_{K V}^{2}\left(\mathfrak{X}(N), C^{\infty}(M)\right)$. We discuss also the geometric interpretation of the class $A \in H_{K V}^{2}\left(\mathfrak{X}(N), C^{\infty}(M)\right)$ for convex locally flat hypersurfaces.

This paper is organized in the following way. Section 2 is devoted to the background on the Koszul-Vinberg cohomology which will be used in the sequel. Section 3 is

Date: Received: February 20, 2012 and Accepted: October 1, 2014.

2010 Mathematics Subject Classification. 53C07, 53C12.

Key words and phrases. KV-cohomology, locally flat hypersurface, scalar second fundamental form, convex manifold, hyperbolic manifold.

The author is supported by Agence Universitaire de la Francophonie and VLIR. 
concerned with the Koszul-Vinberg class of second fundamental forms of locally flat hypersurfaces. In the last section, we give a geometric interpretation of the class $A \in H_{K V}^{2}\left(\mathfrak{X}(N), C^{\infty}(M)\right)$.

\section{Preliminaries on the Koszul-Vinberg cohomology [7]}

Let $\mathbb{F}$ be a commutative field of characteristic zero and $\mathbb{A}$ an algebra over $\mathbb{F}$. The multiplication on the algebra $\mathbb{A}$ is denoted by $a b$ for all $a, b \in \mathbb{A}$.

A left action (resp. a right action )of $\mathbb{A}$ on a $\mathbb{A}$-module $V$ is denoted by av (resp.by $v a$ ) for all $a \in \mathbb{A}$ and for all $v \in V$.

Definition 1.1. The mapp ass : $\mathbb{A}^{3} \rightarrow \mathbb{A}$ defined by ass $(a, b, c)=a(b c)-(a b) c=$ $b(a c)-(b a) c$ is called the associator of $\mathbb{A}$ and $a s s(a, b, c)$, the associator of elements $a, b, c \in \mathbb{A}$.

We say that $\mathbb{A}$ is a Koszul-Vinberg algebra(or a KV-algeba) iff ass $(a, b, c)=$ $a s s(b, a, c)$ for all $a, b, c \in \mathbb{A}$, i.e. the associator of $\mathbb{A}$ is symmetric in the first two entries.

\section{Example 1.1.}

(1) Associative algebras are KV-algebras. Their associators are identically zero.

(2) The vector space $C^{\infty}(\mathbb{R}, \mathbb{R})$ of smooth real-valued functions under the multiplication $f g=f \frac{d g}{d x}$ is a KV-algebra.

(3) Let $(M, \omega)$ be a symplectic manifold of dimension $2 n$. The vector space $\mathfrak{X}(M)$ of smooth vector fields on $M$ is a $\mathrm{KV}$-algebra whose multiplication is defined by the formula $i_{X \diamond Y} \omega=L_{X} i_{Y} \omega$, where $i_{Y} \omega$ is the inner product of the form $\omega$ by the vector field $X$ and $L_{X} \omega$ the Lie derivative of $\omega$ in the direction of $X$.

Definition 1.2. An element $\xi \in \mathbb{A}$ is called a Jacobi element of the $K V$-algebra $\mathbb{A}$ if and only if $a s s(a, b, \xi)=0, \forall a, b \in \mathbb{A}$. The set $J(\mathbb{A})$ of Jacobi elements of $\mathbb{A}$ is an associative subalgebra containing the center of $\mathbb{A}$.

Definition 1.3. We consider a vector space $W$ with two bilinear maps

$$
\mathbb{A} \times W \longrightarrow W:(a, w) \rightarrow a w
$$

and

$$
W \times \mathbb{A} \longrightarrow W:(w, a) \rightarrow w a
$$

We say that $W$ is a two-sided module over $\mathbb{A}$ if and only if for all $a, b \in \mathbb{A}$ and for all $w \in W$ we have $(a b) w-a(b w)=(b a) w-b(a w)$ and $(a w) b-a(w b)=(w a) b-w(a b)$. If the right action (resp. the left action) is trivial, we say that $W$ is a left (resp. a right) $\mathrm{KV}$-module.

The subspace $J(W)$ of the Jacobi elements of the $\mathrm{KV}$-module $W$ is precisely the set of elements $w \in W$ such that ass $(a, b, w)=0$, for all $(a, b) \in W^{2}$.

Example 1.2. Let $V$ and $W$ be two KV-bimodules on the same KV-algebra $\mathbb{A}$. If we denote by $L(W, V)$ the set of all linear maps from $W$ to $V$, then the mappings

$$
\mathbb{A} \times L(W, V) \longrightarrow L(W, V):(a, f) \rightarrow a f
$$

and

$$
L(W, V) \times \mathbb{A} \longrightarrow L(W, V):(f, a) \rightarrow f a
$$


defined by

and

$$
(a f)(w)=a(f(w))-f(a w)
$$

$$
(f a) w=(f(w)) a
$$

are bilinear mappings. Moreover for all $a, b \in \mathbb{A}$ and for all $f \in L(W, V)$ one shows that $(a b) f-a(f b)=(b a) f-b(a f)$ and $(a f) b-a(f b)=(f a) b-f(a b)$. Hence $L(W, V)$ is a $\mathrm{KV}$-bimodule over $\mathbb{A}$ which becomes a left $\mathrm{KV}$-module if $V$ is a left $\mathrm{KV}$-module.

Generally, the vector space $L^{q}(W, V)$ of $q$-linear maps $(q \geq 1)$ from $W$ to $V$ endowed with the actions

$$
(a f)\left(w_{1}, \ldots, w_{q}\right)=a\left(f\left(w_{1}, \ldots, w_{q}\right)\right)-\sum_{j=1}^{q} f\left(w_{1}, \ldots, a w_{j}, \ldots, w_{q}\right)
$$

and

$$
(f a)\left(w_{1}, \ldots, w_{q}\right)=\left(f\left(w_{1}, \ldots, w_{q}\right)\right) a
$$

is a $\mathrm{KV}$-bimodule over $\mathbb{A}$.

Let $\mathbb{A}$ be a $\mathrm{KV}$-algebra and $W$ a $\mathrm{KV}$-bimodule over $\mathbb{A}$. The set $C^{q}(\mathbb{A}, W)$ of all $q$-linear maps from $\mathbb{A}$ to $W$ is a $\mathrm{KV}$-bimodule over $\mathbb{A}$. For all $a \in \mathbb{A}$ and for all integer $1 \leq r \leq q$ we consider the map

where

$$
e_{r}(a): C^{q}(\mathbb{A}, W) \longrightarrow C^{q-1}(\mathbb{A}, W)
$$

$$
\left(e_{r}(a) f\right)\left(a_{1}, \ldots, a_{q-1}\right)=f\left(a_{1}, \ldots, a_{r-1}, a, a_{r}, \ldots, a_{q-1}\right) .
$$

With these notations, it was shown in [6] that the map

$$
d_{K V}: C^{q}(\mathbb{A}, W) \longrightarrow C^{q+1}(\mathbb{A}, W)
$$

defined by

$$
\begin{aligned}
\left(d_{K V} f\right)\left(a_{1}, \ldots, a_{q+1}\right) & =\sum_{1 \leq j \leq q}(-1)^{j}\left(\left(a_{j} f\right)\left(a_{1}, \ldots, \hat{a}_{j}, \ldots, a_{q+1}\right)\right. \\
& \left.+e_{q}\left(a_{j}\right)\left(f a_{q+1}\right)\left(a_{1}, \ldots, \hat{a}_{j}, \ldots, \hat{a}_{q+1}\right)\right)
\end{aligned}
$$

is a coboundary operator, i.e $d_{K V}^{2}=0$.

We set $C(\mathbb{A}, W)=\oplus_{q \geq 1} C^{q}(\mathbb{A}, W)$ and $C^{0}(\mathbb{A}, W)=J(W)$. The map

$$
d_{K V}: C^{0}(\mathbb{A}, W) \longrightarrow C^{1}(\mathbb{A}, W)
$$

defined by $d_{K V} w(a)=-a w+w a$, verifies the relation $d_{K V}^{2}=0$ for all $w \in J(W)$. We then obtain a complete complex

$$
C_{K V}(\mathbb{A}, W)=J(W) \oplus C(\mathbb{A}, W) .
$$

Definition 1.4. The cohomology of the complex $C_{K V}(\mathbb{A}, W)=J(W) \oplus C(\mathbb{A}, W)$ is called the Koszul-Vinberg cohomology or the KV-cohomology.

The $q$-order cohomology space is denoted by $H_{K V}^{q}(\mathbb{A}, W)$.

In particular, if $W$ is a left module, we have : for $q=1$,

is given by

$$
d_{K V}: C^{1}(\mathbb{A}, W) \longrightarrow C^{2}(\mathbb{A}, W)
$$

$$
d_{K V}\left(a_{1}, a_{2}\right)=(-1)^{1}\left(a_{1} f\right)\left(a_{2}\right)=a_{1} f\left(a_{2}\right)-f\left(a_{1} a_{2}\right),
$$


and for $q=2$,

is defined by

$$
d_{K V}: C^{2}(\mathbb{A}, W) \longrightarrow C^{3}(\mathbb{A}, W)
$$

$$
\begin{aligned}
d_{K V}\left(a_{1}, a_{2}, a_{3}\right) & =(-1)^{1}\left(a_{1} f\right)\left(a_{2}, a_{3}\right)+(-1)^{2}\left(a_{2} f\right)\left(a_{1}, a_{3}\right) \\
& =-\left(a_{1} f\left(a_{2}, a_{3}\right)-f\left(a_{1} a_{2}, a_{3}\right)-f\left(a_{1} a_{3}, a_{2}\right)\right) \\
& +\left(-a_{2} f\left(a_{1}, a_{3}\right)-f\left(a_{2} a_{1}, a_{3}\right)-f\left(a_{2} a_{3}, a_{1}\right)\right) \\
& =-a_{1} f\left(a_{2}, a_{3}\right)+f\left(a_{1} a_{2}, a_{3}\right)+f\left(a_{1} a_{3}, a_{2}\right) \\
& +-a_{2} f\left(a_{1}, a_{3}\right)-f\left(a_{2} a_{1}, a_{3}\right)-f\left(a_{2} a_{3}, a_{1}\right) \\
& =-a_{1}\left(f\left(a_{2}, a_{3}\right)\right)+a_{2}\left(f\left(a_{1}, a_{3}\right)\right)+f\left(a_{1} a_{2}-a_{2} a_{1}, a_{3}\right) \\
& +f\left(a_{2}, a_{1} a_{3}\right)-f\left(a_{3}, a_{1} a_{2}\right) \\
& =-a_{1}\left(f\left(a_{2}, a_{3}\right)\right)+a_{2}\left(f\left(a_{1}, a_{3}\right)\right)+f\left(\left[a_{1}, a_{2}\right], a_{3}\right)+ \\
& +f\left(a_{2}, a_{1} a_{3}\right)-f\left(a_{3}, a_{1} a_{2}\right) .
\end{aligned}
$$

Hence, for all $f \in C^{2}(\mathbb{A}, W)$ and for all $a_{1}, a_{2}, a_{3} \in \mathbb{A}$

$$
\begin{aligned}
d_{K V} f\left(a_{1}, a_{2}, a_{3}\right)= & -a_{1}\left(f\left(a_{2}, a_{3}\right)\right)+a_{2}\left(f\left(a_{1}, a_{3}\right)\right)+f\left(\left[a_{1}, a_{2}\right], a_{3}\right) \\
& +f\left(a_{2}, a_{1} a_{3}\right)-f\left(a_{3}, a_{1} a_{2}\right)
\end{aligned}
$$

A 2-cochain $f \in C^{2}(\mathbb{A}, W)$ is a KV-cocycle if and only if for all $a_{1}, a_{2}, a_{3} \in \mathbb{A}$,

$$
f\left(\left[a_{1}, a_{2}\right], a_{3}\right)=a_{1}\left(f\left(a_{2}, a_{3}\right)\right)-a_{2}\left(f\left(a_{1}, a_{3}\right)\right)+f\left(a_{3}, a_{1} a_{2}\right)-f\left(a_{2}, a_{1} a_{3}\right) .
$$

\section{THE ClASS OF THE SCALAR SECOND FUNDAMENTAL FORM}

Definition 2.1. Let $(M, g)$ be a Riemannian manifold, $D$ the Levi-Civita connection of $(M, g)$ and $R$ the curvature tensor of $(M, g, D)$. A submanifold $N$ of $M$ is called a locally flat submanifold if $\tilde{R}(X, Y) Z=0$ for all smooth vector fields $X, Y, Z$ on $N$, where $\tilde{D}$ is the Levi-Civita connection induced by the inclusion of $N$ in $M$ and $\tilde{R}$ the curvature tensor of $\tilde{D}$.

Proposition 2.1. Let $N$ be a locally flat submanifold of $(M, g, D)$. The vector space $\mathfrak{X}(N)$ of smooth vector fields on $N$ is a $K V$-algebra under the multiplication given by

$$
\mathfrak{X}(N) \times \mathfrak{X}(N) \longrightarrow \mathfrak{X}(N):(X, Y) \rightarrow X Y=\tilde{D}_{X} Y .
$$

We denote this algebra by $\mathbb{A}=(\mathfrak{X}(N), \tilde{D})$. Moreover, the vector space $C^{\infty}(M)$ of smooth functions of $M$ is a left $K V$-module over the $K V$-algebra $\mathbb{A}=(\mathfrak{X}(N), \tilde{D})$ under the action $X . f=d f(X)$.

Proof. We will use the flatness of the curvature tensor $\tilde{R}$ and the fact that $\tilde{D}$ is a torsion free connection to show that the associator of the algebra $\mathbb{A}=(\mathfrak{X}(N), \tilde{D})$ is symmetric in the two first entries. For all smooth vector fields on $N$, we have the following equivalent statements:

$$
\begin{aligned}
(X Y) Z-X(Y Z) & =(Y X) Z-Y(X Z) \\
& \Longleftrightarrow \\
\left(\tilde{D}_{X} Y\right) Z-X\left(\tilde{D}_{Y} Z\right) & =\left(\tilde{D}_{Y} X\right) Z-Y\left(\tilde{D}_{X} Z\right) . \\
& \Longleftrightarrow \\
\tilde{D}_{\tilde{D}_{X} Y} Z-\tilde{D}_{X} \tilde{D}_{Y} Z & =\tilde{D}_{\tilde{D}_{Y} X} Z-\tilde{D}_{Y} \tilde{D}_{X} Z \\
& \Longleftrightarrow
\end{aligned}
$$




$$
\begin{aligned}
&\left(\tilde{D}_{\tilde{D}_{X} Y}-\tilde{D}_{\tilde{D}_{Y} X}\right) Z=\left(\tilde{D}_{X} \tilde{D}_{Y}-\tilde{D}_{Y} \tilde{D}_{X}\right) Z \\
& \Longleftrightarrow \\
& \tilde{D}_{\tilde{D}_{X} Y-\tilde{D}_{Y} X} Z=\left[\tilde{D}_{X}, \tilde{D}_{Y}\right] Z .
\end{aligned}
$$

Since $\tilde{D}$ is a torsion-free connection, $\tilde{D}_{X} Y-\tilde{D}_{Y} X=[X, Y]$ and the last equation becomes: $\tilde{D}_{[X, Y]} Z=\left[\tilde{D}_{X}, \tilde{D}_{Y}\right] Z$ or $\tilde{D}_{X} \tilde{D}_{Y} Z-\tilde{D}_{Y} \tilde{D}_{X} Z-\tilde{D}_{[X, Y]} Z=0$, i.e. $\tilde{R}(X, Y, Z)=0$.

\section{Example 2.1.}

(1) A foliation $\mathfrak{F}$ on a smooth manifold $M$ is said to be an affine foliation if the vector space $\mathfrak{X}(\mathfrak{F})$ of smooth vector fields tangent to the leaves of $\mathfrak{F}$ is a $\mathrm{KV}$-algebra whose commutator Lie algebra is a subalgebra of $\mathfrak{X}(M)$. The leaves of an affine foliation are locally flat submanifolds of $M$.

(2) A Lagrangian foliation $\mathfrak{F}$ on a $2 n$-symplectic manifold $(M, \omega)$ is a foliation whose leaves are Lagrangian submanifolds of $M$, i.e., each leaf $F$ is a $n$ dimensional submanifold of $M$ and $\omega(X, Y)=0$ for all vector fields $X, Y$ tangent to the leaves of $\mathfrak{F}$. The symplectic structure of $M$ defines on each leaf $F$ of $\mathfrak{F}$, a connection whose curvature and torsion vanish identically. Therefore, the leaves of a Lagrangian foliation on a symplectic manifold are locally flat submanifolds of $M$.

(3) Let $X \in \mathfrak{X}(M)$ be a smooth vector field on a manifold $M$. The vector space $\mathbb{A}=\left\{f X: f \in C^{\infty}(M)\right\}$ is a KV-algebra under the multiplication $(f X)(g X)=f(d g(X)) X$. The leaves of the flow defined by $X$ are locally flat submanifolds.

We consider a triple $(M, g, D)$ where $(M, g)$ is a Riemannian manifold of dimension $n \geq 2$ and $D$ the metric connection of $(M, g)$. Let $N$ be a $(n-k)$-dimensional submanifold of $M$. We denote by $\tilde{D}$ the metric connection on $N$ induced by the inclusion of $N$ in $M$.

The relationship between the Levi-Civita connection $\tilde{D}$ of the induced metric $\tilde{g}$, the second fundamental form $\vec{A}$ and the ambient Levi-Civita connection $D$ is given by the following Gauss Formula

$$
D_{X} Y=\tilde{D}_{X} Y+\vec{A}(X, Y) .
$$

If $X_{1}, \ldots, X_{k}$ is a local orthogonal frame of vector fields over $N$ in $M$, we can write:

$$
\vec{A}(X, Y)=\sum_{i=1}^{k} A_{i}(X, Y) X_{i}
$$

In particular, if $N$ is a hypersurface, i.e $k=1$ the last formula becomes

$$
\vec{A}(X, Y)=A(X, Y) \xi
$$

where $\xi$ is a local normal unit vector field over $N$ in $M$, i.e a local section of the transversal line bundle.

Definition 2.2. The map $A: \mathfrak{X}(N) \times \mathfrak{X}(N) \longrightarrow C^{\infty}(M):(X, Y) \rightarrow A(X, Y)$ is called the scalar second fundamental form of the hypersurface $N$ in $M$ with respect to the local unit vector field $\xi$.

We have the following 
Proposition 2.2. Let $N$ be a locally flat hypersurface of a Riemannian manifold $(M, g, D)$. The following statements are equivalent.

(1) $R(X, Y) Z \in \mathfrak{X}(N), \forall X, Y \in \mathfrak{X}(N)$.

(2) $R(X, Y) \xi=0, \forall X, Y \in \mathfrak{X}(N)$.

(3) $R(X, \xi) Y=R(Y, \xi) X, \forall X, Y \in \mathfrak{X}(N)$.

Proof. We consider the $(0,4)$-curvature tensor defined by $R(X, Y, Z, W)=$ $g(R(X, Y) Z, W)$ which is symmetric in the first two and the last two entries. For all vector fields $X, Y \in \mathfrak{X}(N)$, we have: $R(X, Y, \xi, Z)=R(Y, X, Z, \xi)$, i.e $g(R(X, Y) \xi, Z)=g(R(Y, X) Z, \xi)$. Hence, $R(X, Y) Z$ is normal to $\xi$, for all $X, Y, Z \in$ $\mathfrak{X}(N)$ if and only if $R(X, Y) \xi$ is identically zero for all $X, Y \in \mathfrak{X}(N)$. This establishes the equivalence between statements (1) and (2).

By the Bianchi's first identity of the $(1,3)$-curvature tensor we have:

$$
R(X, Y) \xi+R(Y, \xi) X+R(\xi, X) Y=0
$$

or

$$
R(X, Y) \xi=-R(Y, \xi) X-R(\xi, X) Y=-R(Y, \xi) X+R(X, \xi) Y .
$$

This establishes the equivalence between statements (2) and (3).

Theorem 2.1. Let $N$ be a locally flat hypersurface of a Riemannian manifold $(M, g, D)$ which satisfies one of the three equivalent statements of the Proposition 3.2. Then the scalar second fundamental form $A$ of $N$ defines a Koszul-Vinberg class $[A] \in H_{K V}^{2}\left(\mathfrak{X}(N), C^{\infty}(M)\right)$.

Proof. We use the Codazzi-Mainardi's formula $D_{X} Y=\tilde{D}_{X} Y+A(X, Y) \xi$. For all vector fields $X, Y, Z \in \mathfrak{X}(N)$, we compute the curvature tensor of $D$

$$
R(X, Y) Z=D_{X} D_{Y} Z-D_{Y} D_{X} Z-D_{[X, Y]} Z \text {. }
$$

using

$$
D_{Y} Z=A(Y, Z) \xi+\tilde{D}_{Y} Z
$$

and

$$
D_{X} Z=A(X, Z) \xi+\tilde{D}_{X} Z
$$

Hence, the curvature tensor of $D$ is given by:

$$
R(X, Y) Z=D_{X}\left(A(Y, Z) \xi+\tilde{D}_{Y} Z\right)-D_{Y}\left(A(X, Z) \xi+\tilde{D}_{X} Z\right)-D_{[X, Y]} Z \text {. }
$$

Therefore,

$$
\begin{aligned}
R(X, Y) Z= & A(Y, Z) D_{X} \xi+X A(Y, Z) \xi+D_{X} \tilde{D}_{Y} Z-A(X, Z) D_{Y} \xi \\
& -Y A(X, Z) \xi-D_{Y} \tilde{D}_{X} Z-A([X, Y], Z) \xi-\tilde{D}_{[X, Y]} Z
\end{aligned}
$$

Setting

$$
E:=D_{X} \tilde{D}_{Y} Z-D_{Y} \tilde{D}_{X} Z-\tilde{D}_{[X, Y]} Z
$$

and

$$
A(X Y, Z):=A\left(\tilde{D}_{X} Y, Z\right)
$$


we have:

$$
\begin{aligned}
E & =A\left(X, \tilde{D}_{Y} Z\right) \xi+\tilde{D}_{X} \tilde{D}_{Y} Z-A\left(Y, \tilde{D}_{X} Z\right) \xi-\tilde{D}_{Y} \tilde{D}_{X} Z-\tilde{D}_{[X, Y]} Z \\
& =A(X, Y Z) \xi-A(Y, X Z) \xi+\tilde{D}_{X} \tilde{D}_{Y} Z-\tilde{D}_{Y} \tilde{D}_{X} Z-\tilde{D}_{[X, Y]} Z \\
& =(A(X, Y Z)-A(Y, X Z)) \xi+\tilde{R}(X, Y) Z \\
& =(A(X, Y Z)-A(Y, X Z)) \xi
\end{aligned}
$$

We obtain finally:

$$
\begin{aligned}
R(X, Y) Z= & A(X, Y) D_{X} \xi+X A(Y, Z) \xi-A(X, Z) D_{Y} \xi-Y A(X, Z) \xi \\
& -A([X, Y], Z) \xi+(A(X, Y Z)-A(Y, X Z)) \xi
\end{aligned}
$$

On the other hand, the normal vector field $\xi$ being a unitary vector field, the vector fields $\xi$ and $D_{X} \xi$ are orthogonal for all smooth vector field $X$ over $N$. Consequently,

$$
\begin{aligned}
R(X, Y, Z, \xi) & =g(R(X, Y) Z, \xi) \\
& =X A(Y, Z)-Y A(X, Z)-A([X, Y], Z) \\
& +A(X, Y Z)-A(Y, X Z) .
\end{aligned}
$$

Thus,

$$
R(X, Y, Z, \xi)=g(R(X, Y) Z, \xi))=d_{K V} A(Y, X, Z)
$$

We see through the last expression, since $g$ is non-degenerate and $\xi$ is non singular, that $d_{K V} A=0$ if and only if $R(X, Y) Z$ is orthogonal to the unitary normal vector field $\xi$.

\section{THE GEOMETRIC INTERPRETATION OF THE SCALAR SECOND FUNDAMENTAL FORM}

We discuss the geometric interpretation of the Koszul-Vinberg class of scalar fundamental forms of convex locally flat hypersurfaces of non flat Riemannian manifolds. We need the following definitions.

Definition 3.1. A submanifold $N$ of a Riemannian manifold $(M, g)$ is said to be convex, if its scalar second fundamental form $A$ is positive definite [10].

Definition 3.2. A Riemannian metric $g$ on a locally flat manifold $(M, D)$ is called a Hessian metric if in a neighborhood $U$ of a point, there exists a local smooth function $\varphi \in C^{\infty}(U)$ such that $g=D^{2} \varphi=D d \varphi$. In affine coordinates $x^{1}, \ldots, x^{n}$ of $(M, D), g\left(\frac{\partial}{\partial x^{i}}, \frac{\partial}{\partial x^{j}}\right)=\frac{\partial^{2} \varphi}{\partial x^{i} \partial x^{j}}$. [9]

Definition 3.3. A locally flat manifold $M$ is said to be a hyperbolic manifold, if the universal covering of $M$ is isomorphic as a flat manifold to a convex open subset of the real affine space which doesn't contain any line. Geodesics of an hyperbolic space are not complete [4], [5] et [6].

We need the following Koszul's Theorem

Theorem 3.1. [4] Let $M$ be a compact locally flat manifold. Then $M$ is a hyperbolic manifold if and only if there exists a closed 1-form $\alpha$ on $M$ such that D $\alpha$ is positive definite in each point of $M$. The first Betti number of a hyperbolic manifold is non zero.

We have the following proposition 
Proposition 3.1. If the $K V$-cohomology class $A \in H_{K V}^{2}\left(\mathfrak{X}(N), C^{\infty}(M)\right)$ of the scalar second fundamental form $A$ of a hypersurface $N$ is trivial, then $A$ defines a de Rham cohomology class $B \in H_{D R}^{1}(N, \mathbb{R})$.

Proof. Suppose that $[A]=0$. There exists a KV 1-cochain $B \in C^{1}\left(\mathbb{A}, C^{\infty}(M)\right)$ such that $A=d_{K V} B$. For all vector fields $X, Y \in \mathfrak{X}(N)$ we have:

$A(X, Y)=-X B(Y)+B(X Y)$. Since $A$ is tensorial we have: $A(f X, Y)=f A(X, Y)$, i.e $-f X B(Y)+B(f X Y)=f(-X B(Y)+B(X Y))$ or $-f X B(Y)+B(f X Y)=$ $-f X B(Y)+f B(X Y)$. Therefore, $B(f X Y)=f(B X Y)$ and hence, $B$ is tensorial. Since the 2-cochain $A$ is symmetric, we have: $-X B(Y)+B(X Y)=A(X, Y)=$ $A(Y, X)=-Y B(X)+B(Y X)$ and

$$
X B(Y)-Y B(X)-B([X, Y])=0 .
$$

We see through the last expression that the 1-cochain $B$ is a de Rham closed 1-form on the hypersurface $N$. The closed form $B$ determines a de Rham cohomology class $B \in H_{D R}^{1}(N, \mathbb{R})$.

Suppose that the class $B \in H_{D R}^{1}(N, \mathbb{R})$ defined above is trivial and $N$ is a compact hypersurface of $M$. There exists a smooth function $f \in C^{\infty}(N)$ such that $B=d f$, and hence $A=D^{2} f$. The locally flat manifold $N$ is equipped with a globally Hessian metric induced by its second fundamental form. Since the hypersurface $N$ is a compact manifold, $f$ possess critical points, consequently, the signature of the Hessian of $f$ is not constant. On the other hand, if the Euler characteristic of the compact hypersurface $N$ is non zero, since its first Betti number is non zero, then $B$ defines a hyperfoliation $F$ on $N$. The foliation $F$ is Riemannian since $B$ is a closed de Rham 1-form. We conclude this discussion with the following theorem.

Theorem 3.2. Let $N$ be a convex locally flat manifold such that one of the equivalent statements of Proposition 3.2 holds.

We suppose that the class $A \in H_{K V}^{2}\left(\mathfrak{X}(N), C^{\infty}(M)\right)$ is trivial. We then have the following geometric information:

(1) The hypersurface $N$ is compact if and only if it is hyperbolic.

(2) The following statements are mutually exclusive: $B \in H_{D R}^{1}(N, \mathbb{R})=0$ and $N$ is a compact hypersurface of $M$.

(3) If the Euler characteristic $\mathfrak{X}(N)$ of $N$ is non zero, then the scalar second fundamental form $A$ define (through B) a Riemannian hyperfoliation on $N$.

\section{REFERENCES}

[1] Defever, F.; Deszcz, R.; Verstraelen, L. and Yaprak,S., Pseudosymmetry type curvature properties of hypersurfaces, Proceedings of the Conferences on Differential Geometry and Vision, Theory of Submanifolds, (1992), 109-131.

[2] Delanoe, Ph., Remarques sur les variétés localement hessiennes, Osaka J. Math. 26 (1989), 65-69.

[3] Duggal, Krishan L. and Bejancu, A., Lightlike Submanifolds of Semi-Riemannian Manifolds and Applications, Kluwer Academic Publishers, Dordrecht, 1996.

[4] Koszul, J.L, Variétés locament plates et convexité, Osaka J. Math. 2 (1965), 285-290.

[5] Koszul, J.L, Déformations des connexions localement plates, Ann.Inst.Fourrier 18 (1968), 103-114. 
[6] Koszul, J.L, Connexions hyperboliques et déformations, Sympos. Math. 11 (1968), 357-361.

[7] Nguiffo Boyom, M., The Cohomology of Koszul-Vinberg Algebras, Pacific Journal of Mathematics, 225 (2005), no. 1, 101-134.

[8] Nguiffo Boyom, M., Some Lagrangian invariants of symplectic manifolds, Banach Center Publications, 74 (2007).

[9] Nguiffo Boyom, M. and Wolak, R., Local structure Koszul-Vinberg and Lie algebroids, Bull. Sci. Math 2 (2004), no. 6, 467-479.

[10] Shima, H., The geometry of Hessian structures, World Scientific Publishing, 2007.

\section{Acknowledgements}

I would like thank Professor Michel Nguiffo Boyom of University of Montpellier for his kindness and wise advise during my postdoc fellowship at Montpellier.

Département de Mathématiques et Informatique, Faculté des Sciences, Université de Kinshasa, B.P. 190 Kinshasa Xi, Rép. Dém. Congo and Centre de Recherche sur L'Enseignement de la Mathématique (CREM) Kinshasa-Gombe.

E-mail address: musesalanda@yahoo.fr 